\title{
A dual brain-targeting curcumin-loaded polymersomes ameliorated cognitive dysfunction in intrahippocampal amyloid- $\beta_{1-42}$-injected mice
}

This article was published in the following Dove Press journal:

International Journal of Nanomedicine

5 August 2016

Number of times this article has been viewed

\author{
Tingting Jia ${ }^{1, *}$ \\ Zhiguo Sun ${ }^{1, *}$ \\ Ying Lu' \\ Jie Gao' \\ Hao Zou' \\ Fangyuan $\mathrm{Xie}^{2}$ \\ Guoqing Zhang ${ }^{2}$ \\ $\mathrm{HaO} \mathrm{Xu}^{3}$ \\ Duxin Sun ${ }^{3}$ \\ Yuan Yu' \\ Yanqiang Zhong' \\ 'Department of Pharmaceutical \\ Sciences, School of Pharmacy, The \\ Second Military Medical University, \\ ${ }^{2}$ Department of Pharmacy, Eastern \\ Hepatobiliary Surgery Hospital, \\ Shanghai, People's Republic of China; \\ ${ }^{3}$ Department of Pharmaceutical \\ Sciences, School of Pharmacy, \\ University of Michigan, Ann Arbor, \\ MI, USA \\ *These authors contributed equally \\ to this work
}

Correspondence: Yuan Yu;

Yanqiang Zhong

Department of Pharmaceutical Sciences,

School of Pharmacy, The Second Military

Medical University, 325 Guohe Road,

Shanghai 200433, People's Republic

of China

Tel/fax +86 2I 8I87 I285

Email pharmyuu@।63.com;

yqzhong68@163.com

\begin{abstract}
Due to the impermeability of the blood-brain barrier and the nonselective distribution of drugs in the brain, the therapeutic access to intractable neurological disorders is challenging. In this study, dual brain-targeting polymersomes (POs) functionalized by transferrin and Tet-1 peptide (Tf/Tet-1-POs) promoted the transportation of curcumin into the brain and provided neuroprotection. The modification of the ligands that bind to the surface of POs was revealed by $\mathrm{X}$-ray photoelectron spectroscopy analysis. The cell uptake of a coculture model of mouse brain capillary endothelial cells with neurons showed that the Tf/Tet-1-POs had significant transportation properties and possessed affinity for neurons. The pharmacokinetic analysis showed that the blood-brain barrier permeability-surface efficiency of the Tf/Tet-1-POs was $0.28 \mathrm{~mL} / \mathrm{h} / \mathrm{g}$ and that the brain tissue uptake rate $(\% \mathrm{ID} / \mathrm{g}$ ) was 0.08 , which were significant compared with the controls $(P<0.05)$. The curcumin-encapsulated Tf/Tet-1-POs provided neuroprotection and ameliorated cognitive dysfunction in intrahippocampal amyloid- $\beta_{1-42}$-injected mice. These results suggest that the dual brain-targeting POs are more capable of drug delivery to the brain that can be exploited as a multiple noninvasive vehicle for targeting therapeutics.
\end{abstract}

Keywords: polymersomes, transferrin, Tet-1 peptide, Alzheimer's disease

\section{Introduction}

Liposomes have been extensively studied as drug carriers but are of limited use due to the disadvantages of frangibility to oxidation and less stability. As a novel drug carrier with a bimolecular membrane structure similar to liposomes, polymersomes (POs) are formed by self-assembly of amphipathic block copolymers, composed of thermodynamically incompatible chain segments. Therefore, copolymers tend to form supramolecular aggregates with specific shapes. ${ }^{1-3}$ In addition, the physicochemical properties and in vivo behavior of POs can be regulated by selecting the type, molecular weight, and proportions of the block polymers. ${ }^{2}$ Characterized by high stability, nano-size homogenicity, and ability to undergo chemical modification, POs can be exploited as a promising noninvasive delivery system for the encapsulation and controlled release of guest molecules.

Neurodegenerative diseases have complex disease mechanisms and represent a severe challenge in the field of neuroscience. Nearly 35.6 million patients worldwide are suffering from dementia since 2012, and 70\% of these cases are attributed to Alzheimer's disease (AD). ${ }^{4}$ The symptoms of AD include progressive memory loss, cognitive dysfunction, and changes in personality, behaviors, and emotional control. A $\beta$ protein deposition is a key pathological feature of $\mathrm{AD}$; therefore, the key strategy for 
the treatment of $\mathrm{AD}$ is to fight the deposition of $\mathrm{A} \beta$ and resist their neurotoxic effects. ${ }^{5}$ Curcumin, a polyphenol extracted from the rhizome of Curcuma longa, has a wide range of biological and pharmacological activities. ${ }^{6}$ It has been found that curcumin can bind to $A \beta$, and promote degradation of deposited plaques and reduce $A \beta$-induced neurotoxicity. ${ }^{7,8}$ Ono et al proved that curcumin binds to amyloid- $\beta_{40}$ and amyloid- $\beta_{42}$ in a dose-dependent manner, inhibiting further aggregation of amyloid- $\beta_{40}$ and amyloid- $\beta_{42}{ }^{9}$ However, curcumin has poor solubility and is unstable in the neutral and alkaline conditions. Studies indicated that curcumin has poor absorption and undergoes rapid metabolism so that its bioavailability is curtailed severely when administered via oral or intravenous (iv) route. ${ }^{10}$ Therefore, some studies have tried to encapsulate curcumin in nanoparticles and liposomes for treating multidrug-resistant tumors. ${ }^{10,11}$

The blood-brain barrier (BBB) is a special restrictive biochemical barrier characterized by tight junctions within the brain capillary endothelial cells (BCECs), which prevents the uptake of most pharmaceuticals by the brain. ${ }^{12}$ Therefore, the nano-vehicles that can be mediated by receptors and actively transport drugs into the brain have been investigated. ${ }^{13}$ Transferrin (Tf) is an iron-binding blood plasma glycoprotein, and the Tf receptors (TfRs) are present on the membrane of BCECs. In addition to brain endothelial cells, TfRs are also found on the plasma membrane of neurons. ${ }^{14}$ Pang et al showed that the uptake of Tf-conjugated POs occurred mainly through a clathrin-mediated energy-dependent endocytosis, and also showed enhanced intracellular delivery and chemotherapy in rats with glioma. ${ }^{15}$ The tetanus toxin has a high affinity for neurons, as its binding is mediated exclusively by ganglioside GT1B receptor on the neurons. ${ }^{16}$ It has been confirmed that tetanus toxin-modified nanoparticles are endocytosed by neurons via receptor internalization. ${ }^{17}$ The phage display test revealed that a 12-amino acid peptide, Tet-1, possesses a high affinity for GT1B receptor. ${ }^{18,19}$ When plasmid DNA encoding luciferase was condensed with Tet1-polyethylene glycol (PEG)-polyethyleneimine and delivered via intracerebroventricular injection to mice, the GT1B-positive stain of transfected nerve cells was observed after delivery of Tet1-mediated vector (58\%) as compared to untargeted vector $(13 \%){ }^{18}$

In most studies, two ligands modified the nanoparticles were used for cancer therapy; these ligands identified the receptors on the tumor cells so as to achieve a sequential targeting action. Bhattacharyya et $\mathrm{al}^{20}$ developed a gold nanoparticle functionalized by EGF receptor and folate antibody for the treatment of ovarian cancer in which the cancer cells expressed EGF receptor and folate receptor. In Gao et al's research, PEG-polycaprolactone nanoparticles modified with angiopep-2 peptide and EGFP-EGF1 protein for drug delivery to neuroglial cells by virtue of angiopep-2 crossed the BBB and EGFP-EGF1 bound to the neuroglial cells. ${ }^{21}$

Aiming to improve the brain-targeting efficiency of nanocarrier systems, PEG-poly(D,L-lactic-co-glycolic acid) (PLGA) POs were prepared by a self-assembly method and loaded with curcumin. Moreover, POs were functionalized by $\mathrm{Tf}$ and Tet-1 peptide, forming a dual-ligand-modified drug delivery system (Tf/Tet-1-POs) capable of promoting two stages of brain targeting. Tf/Tet-1-POs crossed the BBB by $\mathrm{Tf}$-mediated endocytosis, and were further delivered to neurons due to the affinity between Tet- 1 and GT1B receptor. Curcumin was encapsulated in Tf/Tet-1-POs, and provided neuroprotection and ameliorated cognitive dysfunction in intrahippocampal amyloid- $\beta_{1-42}$-injected mice.

\section{Materials and methods Materials and animals}

Tf, amyloid- $\beta_{1-42}$, and 6-coumarin were purchased from SigmaAldrich Co. (St Louis, MO, USA). Curcumin (purity 99.5\%) was purchased from Dalian Meilun Biology Technology Ltd. (Dalian, People's Republic of China). D,L-Lactide and glycolide were obtained from PURAC (Gorinchem, the Netherlands). Maleimide-PEG (molecular weight [MW] $3,500 \mathrm{Da}$ ) was purchased from Jenkem Technology Corporation (Beijing, People's Republic of China). 1,1'-Dioctadecyl3,3,3',3'-tetramethylindotricarbocyanine iodide (DiR) dye was purchased from AAT Bioquest Inc. (Sunnyvale, CA, USA). All the other chemicals were of analytical reagent grade and used without further purification. BALB/c mice (male, weighing 20-22 g), nude mice (male, weighing 18-20 g), and C57BL/6 mice (male, weighing 20-22 g) were obtained from Shanghai SLAC Laboratory Animal Co. Ltd. (Shanghai, People's Republic of China). The Neuro-2a cell line was obtained from ATCC (Manassas, VA, USA). The mouse brain capillary endothelial cell line (bEnd.3) was a gift from Professor Yaocheng Rui (Department of Pharmacology, The Second Military Medical University, People's Republic of China).

All animal experiments were approved by the Ethical Committee of the Second Military Medical University. Animals were housed and fed according to the guidelines set by the Second Military University.

\section{Copolymer synthesis and Tf/Tet-I-POs preparation}

As mentioned, ${ }^{22}$ the copolymer of maleimide-PEG-PLGA was formed by ring-opening polymerization of $\mathrm{D}, \mathrm{L}-$ lactide 
and glycolide using maleimide-PEG (MW 3,500 Da) with the terminal hydroxyl groups as initiator in the presence of stannous octoate as catalyst. The MW and the composition of copolymer were identified using ${ }^{1} \mathrm{H}$ NMR and ${ }^{13} \mathrm{C}$ NMR spectroscopy.

The POs were obtained by a self-assembly process as follows: $5 \mathrm{mg}$ of maleimide-PEG-PLGA was dissolved in $5 \mathrm{~mL}$ of acetone, and then dripped into $5 \mathrm{~mL}$ of phosphatebuffered saline (PBS; 0.01 M, pH 6.0) and stirred for 4 hours gently. The POs were obtained after the organic solvent was removed via vacuum-rotary evaporation procedure. Tet-1 peptide was dissolved in deionized water and added to the POs suspension. Tet-1-POs were obtained after 4 hours of reaction at room temperature under nitrogen atmosphere. The Tf was dissolved in PBS (0.15 M, pH 8.5) and mixed with 2-iminothiolane at a 40:1 molar ratio of Tf:2-iminothiolane, and then thiolated-Tf was obtained. The thiolated-Tf was added to Tet-1/POs, followed by 6 hours of slow reaction at room temperature with nitrogen. Tf/Tet-1-POs loaded with 6-coumarin, curcumin, or DiR were prepared as mentioned, but the encapsulated ingredients were added to acetone and dissolved together with the maleimide-PEG-PLGA. Following loading, Tf/Tet-1-POs were purified using the Sepharose CL-4B gel column.

\section{Tf/Tet-I-POs characterizations}

The particle size and zeta potential value of POs were identified with a 3600 Nano ZS Zetasizer (Malvern Instruments, Malvern, UK). The morphology of vesicles was observed by a JEOL 2010 transmission electron microscope (JEOL, Tokyo, Japan). To analyze the linkage of ligands on the surface of POs and the formation of hydro-layer by PEG outside the membrane, X-ray photoelectron spectroscopy (XPS) analysis was applied to related elemental surface compositions of carbon (C1s), oxygen (O1s), and nitrogen (N1s) of the POs, Tf-POs, Tet-1-POs, and Tf/Tet-1-POs as previously described (PHI-5000C ESCA System; Physical Electronics Inc., Eden Prairie, MN, USA). AugerScan3.21 software was used for the data analysis.

\section{In vivo imaging of Tf/Tet-I-POs}

The fluorescent probe Dir-labeled POs were prepared, which included four sample groups: DiR-POs, DiR-Tf-POs, DiRTet-1-POs, and DiR-Tf/Tet-1-POs. Nude mice were injected via caudal vein $(0.5 \mathrm{mg} / \mathrm{kg})$. After 4 hours of administration, the mice received intraperitoneal injections of $100 \mu \mathrm{L}$ of $2 \%$ sodium pentobarbital for anesthesia, and the distribution of fluorescence was observed by an IVIS ${ }^{\circledR}$ Spectrum in vivo imaging system (Xenogen Corp., Alameda, CA, USA).
Subsequently, the animals were sacrificed, and the brain tissue was imaged to observe the fluorescence intensity. ${ }^{23,24}$

\section{In vitro cell uptake of Tf/Tet-I-POs}

A stable, non-pH-dependent fluorescent indicator, 6-coumarin, was entrapped in POs for sample preparation, including 6-coumarin-POs, 6-coumarin-Tf-POs, 6-coumarinTet-1-POs, and 6-coumarin-Tf/Tet-1-POs groups. The bEnd. 3 cells and Neuro-2a cells were used to evaluate the brain-targeting characteristics of Tf/Tet-1-POs. The two kinds of cells were cultured on polylysine-coated coverslips at a density of $10^{4} / \mathrm{cm}^{2}$ for 3 days in confocal plates. The samples described (6-coumarin, $2 \mathrm{ng} /$ well) were added and incubated for 2 hours at $37^{\circ} \mathrm{C}$. The cellular uptakes of samples were observed by the confocal laser scanning microscope (TCS SP5; Leica Microsystems, Wetzlar, Germany).

The bEnd. 3 cells were cultured in the upper chamber of a Transwell ${ }^{\circledR}$ cell culture insert (Corning Incorporated, Corning, NY, USA) at a density of $5 \times 10^{5} / \mathrm{cm}^{2}$. Meanwhile, Neuro-2a cells were cultured in the lower chamber. The cell uptake experiments were conducted when the transepithelial electrical resistance was stabilized at $150 \Omega \times \mathrm{cm}^{2}$ for 3 consecutive days. ${ }^{25}$ The samples ( $4 \mathrm{ng} /$ well) were added to the upper chamber of the Transwell. At 2 hours after drug administration, the Neuro-2a cell uptakes were analyzed by a high-performance liquid chromatography (HPLC) system (LC-20A; Shimadzu, Kyoto, Japan). Using acetic acid ( $\mathrm{pH} 4.0$ )/acetonitrile solution $(5: 95, \mathrm{v} / \mathrm{v})$ as the mobile phase at a flow rate of $1.0 \mathrm{~mL} / \mathrm{min}$, chromatographic separations were achieved with a Dikma Diamonsil ${ }^{\circledR} \mathrm{C} 18(5 \mu \mathrm{m}, 200 \times 4.6 \mathrm{~mm})$ column at $35^{\circ} \mathrm{C}$. The fluorescence detector wavelength was set at Ex $465 \mathrm{~nm} / \mathrm{Em}$ $502 \mathrm{~nm}$. The protein concentrations of Neuro-2a cells were evaluated by the BCA protein assay kit (Thermo Fisher Scientific, Waltham, MA, USA). Uptake per milligram of cellular protein (uptake index, $\mathrm{ng} / \mathrm{mg}$ ) was calculated as the concentrations of 6-coumarin divided by the corresponding concentrations of cellular protein.

\section{Pharmacokinetics}

The turbidity method combined with HPLC was used for drug loading and release of POs in vitro. ${ }^{22}$ A precise amount of curcumin-POs $(1.0 \mathrm{~mL}$ ) was placed in a dialysis bag (MW $10,000 \mathrm{Da})$ and incubated at $37^{\circ} \mathrm{C}(100 \mathrm{rpm})$. The release medium (pH 5.0) was $25 \mathrm{~mL}$ of $0.9 \% \mathrm{NaCl}$ solution containing $50 \%$ ethanol $(95: 5, \mathrm{v} / \mathrm{v}) .{ }^{26}$ At different time points, $0.1 \mathrm{~mL}$ of samples was measured, and then the amount of drug loaded into POs and the in vitro cumulative release rate were calculated.

For pharmacokinetic study, different experimental formulations with different ligands, curcumin-POs, 
curcumin-Tf-POs, curcumin-Tet-1-POs, and curcumin-Tf/ Tet-1-POs, were prepared. BALB/c mice were randomly divided into four groups and administrated a dose of $0.5 \mathrm{~mL}$ (5 mg/kg) via cauda vein. At 2, 5, 15, 30, and 45 minutes and $1,6,12$, and 24 hours, $100 \mu \mathrm{L}$ of separated plasma was collected and mixed with $10 \mu \mathrm{L}$ of emodin $(10 \mu \mathrm{g} / \mathrm{mL})$ as an internal standard. After mixing for 10 seconds, $500 \mu \mathrm{L}$ of ethyl acetate was added and then vortexed for extracting drug. The upper organic solution was then dried under nitrogen and dissolved in $100 \mu \mathrm{L}$ of acetonitrile. Twenty microliters of the supernatant was determined by the HPLC system (LC-20A; Shimadzu) which was equipped with a Dikma Diamonsil ${ }^{\circledR}$ $\mathrm{C} 18(5 \mu \mathrm{m}, 250 \times 4.6 \mathrm{~mm})$ column at $35^{\circ} \mathrm{C}$. The mobile phase was composed of acetonitrile $/ 5 \%$ acetic acid solution $(60: 40, \mathrm{v} / \mathrm{v})$ and was used at a flow rate of $1.0 \mathrm{~mL} / \mathrm{min}$. The fluorescence detector wavelength was set at Ex $436 \mathrm{~nm} / \mathrm{Em}$ $518 \mathrm{~nm} .{ }^{8,27}$ After blood samples were taken at 2, 5, 15, 30, 45, and 60 minutes, the animals were sacrificed for removal of the brain. The brain tissue was removed and homogenized. After adding $10 \mu \mathrm{L}$ of the internal standard emodin $(10 \mu \mathrm{g} / \mathrm{mL})$, the extraction of drugs was performed in the same way as plasma preparation. Twenty microliters of the sample was injected into the HPLC system to determine the concentrations of curcumin in the brain tissue. Analysis of the data was executed using noncompartmental model and statistics momental analysis, from which pharmacokinetic parameters were evaluated. The brain tissue volume of drug distribution $\left(V_{\mathrm{D}}\right)$ was calculated using the ratio of drug in the brain ( $\% \mathrm{ID} / \mathrm{g}$ ) divided by the terminal concentrations of drug in the blood $(\% \mathrm{ID} / \mathrm{mL}){ }^{28,29}$ The brain permeability-surface area $(P S)$ product was calculated using the following formula:

$$
\mathrm{PS}=\frac{\left[V_{D}-V_{0}\right] \mathrm{Cp}(T)}{\int_{0}^{t} \mathrm{Cp}(t) \mathrm{dt}},
$$

where $C p(T)$ is the terminal blood concentration of drug and $V_{0}$ is the brain volume distribution of a blood volume marker. ${ }^{28}$ As described, ${ }^{28,29}$ the uptake by brain tissue was indicated as $\% \mathrm{ID} / \mathrm{g}$ and calculated using the following formula:

$$
\% \mathrm{ID} / \mathrm{g}(t)=\mathrm{PS} \times \mathrm{AUC}_{0-\mathrm{t}} .
$$

\section{Tf/Tet-I-POs loaded with curcumin ameliorated cognitive dysfunction induced by amyloid- $\beta_{1-42}$}

Amyloid- $\beta_{1-42}$ was dissolved in sterile saline solution $(1.0 \mathrm{~g} / \mathrm{L})$, followed by aggregation via incubation at $37^{\circ} \mathrm{C}$ for 96 hours. ${ }^{30} \mathrm{C} 57 \mathrm{BL} / 6$ mice were anesthetized with $10 \%$ chloral hydrate via intraperitoneal injection $(250 \mathrm{mg} / \mathrm{kg})$, and then mounted in a stereotaxic apparatus (RWD Life Science, Shenzhen, People's Republic of China). A midline incision was made on top of skull in order to expose the bregma that is seen as the original point. Injections were done bilaterally into the lateral ventricles, with $1 \mu \mathrm{L}$ of condensed amyloid- $\beta_{1-42}$ $(2 \mathrm{~g} / \mathrm{L})$ per side at the rate of $0.5 \mu \mathrm{L} / \mathrm{min}(1.9 \mathrm{~mm}$ anteroposterior, $\pm 1.7 \mathrm{~mm}$ mediolateral, and $-1.9 \mathrm{~mm}$ dorsoventral to the bregma). ${ }^{30-32}$ The incision was sterilized and stitched. The sham control group underwent the same way of injection with physiological saline.

At 14 days postoperation, the mice were randomly divided into seven groups $(n=8)$. The experimental groups were administrated by caudal vein the following formulations $(15 \mathrm{mg} / \mathrm{kg})$ : curcumin solution (suspended in $1 \%$ sodium carboxymethyl cellulose solution), curcumin-POs, curcumin-Tf/-POs, curcumin-Tet-1-POs, and curcumin-Tf/ Tet-1-POs. The AD control group and the sham control group were injected with saline. After 14 days of consecutive administration, the behavioral changes of the animals were tested using a Morris water maze experimental installation and recorded with a video camera and analyzed with DigBehv software (Jiliang Software Technology Co., Ltd., Shanghai, People's Republic of China). As described, ${ }^{33}$ the training for the behavioral test was carried out during the first 5 days; the animals were released into a circular pool with a diameter of $150 \mathrm{~cm}$ and a depth of $50 \mathrm{~cm}$ that was divided equally into four quadrants. A $10 \mathrm{~cm}$ diameter platform was placed in the second quadrant, and then the pool was filled with water until the platform is $1 \mathrm{~cm}$ under the water surface. Titanium dioxide was applied so that the water appeared opaque. The mice were put into four quadrants in a random order. The time that the mice took to find the platform was recorded, with a cutoff time of 60 seconds. If the mice did not find the platform, they were led to the platform and made to stay on it for 15 seconds, recording latency times as 60 seconds. Both the latency to arrive at the platform and the significant decline in latency over the training days can be used for describing cognitive functions of the animals. The mice were subjected to the probe test on the sixth day. The animals were released into the water twice far away from the position where the platform was removed. The frequency of platform-site crossovers of animals was recorded and analyzed. The objective of the probe trial was to ensure that its spatial preference is a reflection of the memory of the goal location. ${ }^{34}$ Thus, we evaluated the memory and behavior improvement of the mice that could be attributed to the drug delivery system. 
All data were represented as mean \pm standard error of the mean. Statistical analysis was performed using one-way analysis of variance, and $t$-test was used for comparisons between two groups. $P$-value $<0.05$ was considered statistically significant. All of the statistical analyses were performed by using SPSS 11.5 software.

\section{Results and discussion}

\section{The preparation and characterization of Tf/Tet-I-POs}

The number-average molecular weight and the composition of block copolymers were identified and calculated using ${ }^{1} \mathrm{H}$ NMR and ${ }^{13} \mathrm{C}$ NMR spectroscopy (Figure 1) as described before. ${ }^{22}$ The average molecular weight ratio of maleimide-PEG to PLGA was calculated to be 3,500:10,148. The LA/GA composition was 50:50. The dynamic light scattering results showed that the average sizes of POs and $\mathrm{Tf} /$ Tet-1-POs were $50.4 \pm 4.6$ and $63.2 \pm 8.7 \mathrm{~nm}$. The zeta potential values of POs and Tf/Tet-1-POs were $-6.2 \pm 0.08$ and $-5.3 \pm 0.19 \mathrm{mV}$. As shown in Figure 2, transmission electron microscopy revealed that the structure of POs possessed an outer membrane layer.

The blocks of the amphiphilic copolymers are generally thermodynamically incompatible, which leads to the trend of forming supramolecular aggregates via self-assembly process. The bilayer membrane of POs, similar to biofilms structure, is formed by self-assembly. ${ }^{1-3}$ Therefore, POs have potential applications in the field of packaging and controlled release of guest molecules. Since the volume fraction of the hydrophilic chains of copolymers is in the range of $0.20 \%-0.42 \%$ of the total volume of molecules, they tend to form hollow vesicles with membrane wall-like structure. In this study, the POs vector was prepared using block copolymers PEG-PLGA, the membranes of which are difficult to oxidize; therefore, the stability is greatly enhanced. The properties of POs could be further controlled by modulating the properties of the polymers, which includes molecular structure, molecular weight, and ratio of hydrophilic/hydrophobic chain segments. In addition, the POs offer advantages such as requirement of moderate condition for preparation and reduced effects on the drug activities. ${ }^{2}$ Brain-targeting system modified with two different ligands has also been developed and offers the advantage of dual functionality. Therefore, the modification of ligands on the surface of vectors is crucial to improve the targeting effect.

XPS was used to analyze the element compositions, 5-10 nm below the surface of the POs, thus revealing the ligands covalently bonded with POs. The surface chemical compositions of carbon (C1s), oxygen (O1s), and nitrogen (N1s) on the surface of different ligands-modified POs are displayed in Table 1. The peaks at binding energies of 281-307, 527-545, and 398-414eV were ascribed to the elements $\mathrm{C} 1 \mathrm{~s}, \mathrm{O} 1 \mathrm{~s}$, and N1s, respectively. The XPS survey scans are elucidated in Figure 3. The curve-fitting results showed that the $\mathrm{C} 1 \mathrm{~s}$ regions were presented by four peaks (Figure 3A-D), of which the peak 1 signal represented $\mathrm{C}-\mathrm{C} / \mathrm{C}-\mathrm{H}(285.1 \mathrm{eV})$, the peak 2 signal represented $\mathrm{C}-\mathrm{O}-\mathrm{C}$ $(286.4 \mathrm{eV})$, the peak 3 signal represented $\mathrm{O}=\mathrm{C}-\mathrm{C} *(-\mathrm{C})-\mathrm{O}$ $(287.6 \mathrm{eV})$, and peak 4 signal corresponded to $\mathrm{O}-\mathrm{C}=\mathrm{O}$ $(289.1 \mathrm{eV})$. The O1s regions could be presented by peak signals that corresponded to $\mathrm{O}=\mathrm{C}$ (peak $5,532.6 \mathrm{eV}$ ) and $\mathrm{O}-\mathrm{C}$ (peak 6, $533.4 \mathrm{eV}$ ) (Figure 3E-H). The N1s generated by Tf-POs, Tet-1-POs, and Tf/Tet-1-POs could be corresponded to peak $7(400.6 \mathrm{eV}$ ) with a weak signal (Figure $3 \mathrm{~J}-\mathrm{L})$, which displayed percentage values as $0.46 \%, 0.67 \%$, and $0.90 \%$, respectively. The POs had no existence of N1s. Comparison
A

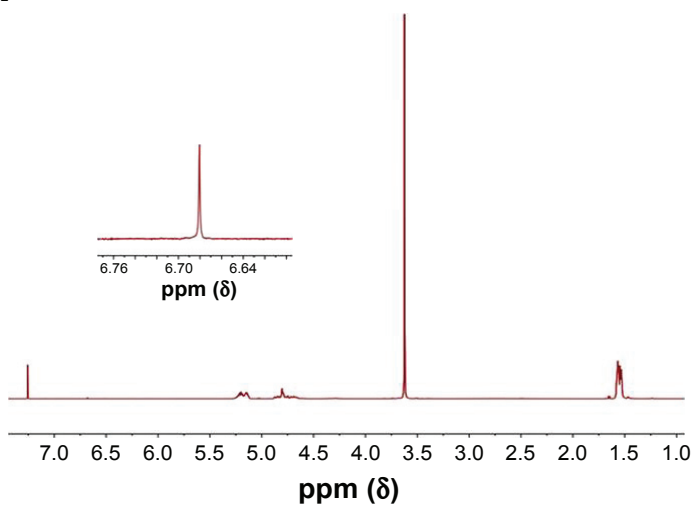

B

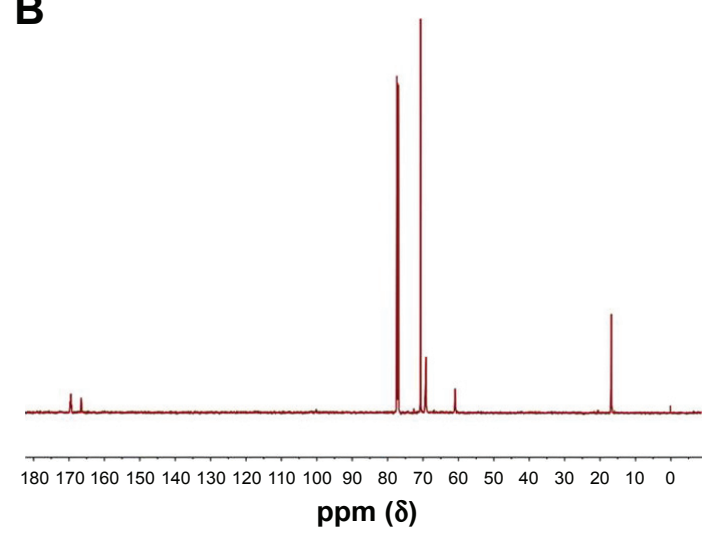

Figure I NMR spectrum of maleimide-PEG-PLGA copolymer in $\mathrm{CDCl} 3$.

Notes: (A) 'H NMR spectrum and (B) ${ }^{13} \mathrm{C}$ NMR spectrum of maleimide-PEG-PLGA copolymer in $\mathrm{CDCl}_{3}$.

Abbreviations: PEG, poly(ethylene glycol); PLGA, poly(D,L-lactic-co-glycolic acid); Ppm, part per million. 

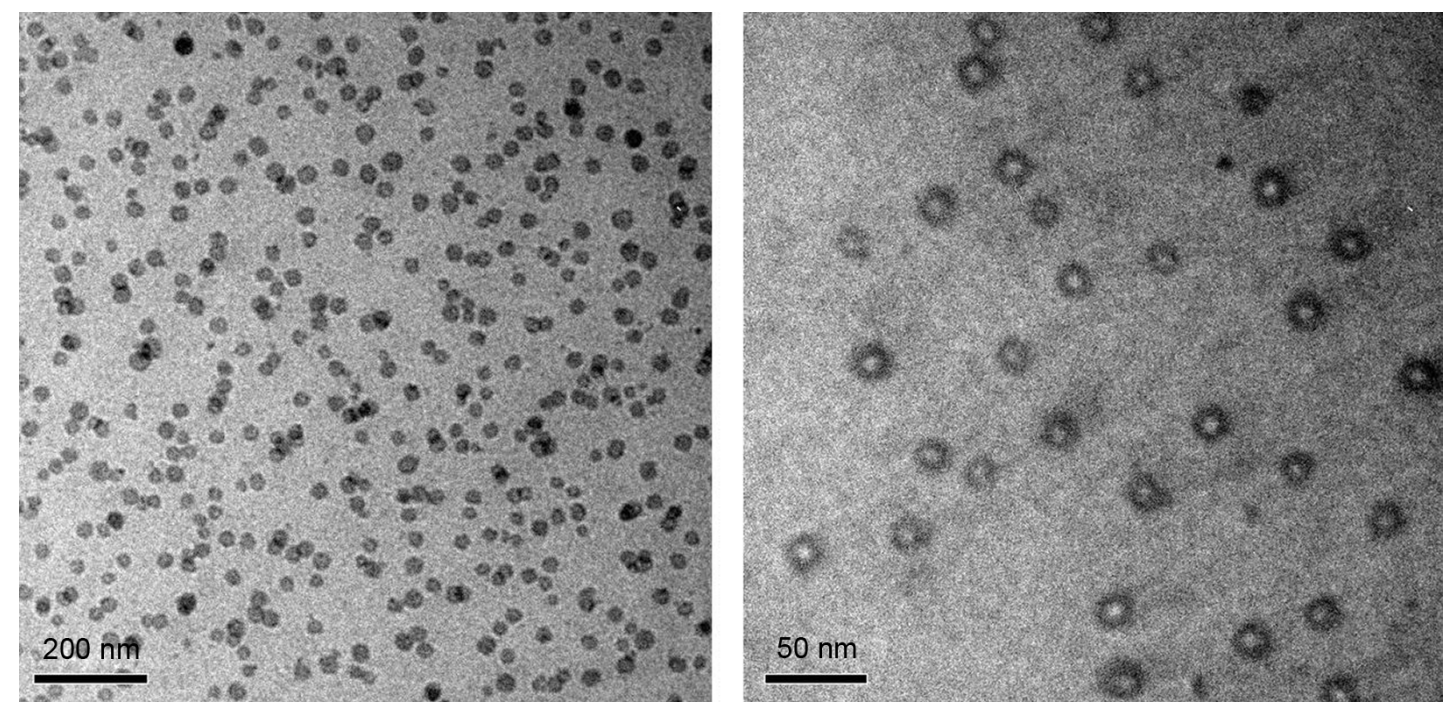

Figure 2 TEM images of Tf/Tet-I-POs (bar 200 and $50 \mathrm{~nm}$ ).

Abbreviations: TEM, transmission electron microscopy; Tf, transferrin; POs, polymersomes.

of the abundance of $\mathrm{C}-\mathrm{O}-\mathrm{C}$ at the binding energy of $286.4 \mathrm{eV}$ showed that the peak ratios for POs, Tet-1-POs, Tf-POs, and Tf/Tet-1-POs were $23.3 \%, 23.6 \%, 25.9 \%$, and $25.2 \%$, respectively; it was suggested that the PEG layer existed at outer layers of POs. XPS can be used to measure the energy spectrum of the outer orbital electron transition of elements; therefore, the structural state of the chemical bonds is reflected. For the chemical structures, the surface N1s of POs was ascribed to the maleimide group in maleimide-PEG, $\mathrm{Tf}$, or Tet-1. As described before, the N1s expression in the maleimide group cannot be detected by XPS. The N1s in the Tf/Tet-1-POs accounted for $0.9 \%$ of all carbon, oxygen, and nitrogen elementary compositions, which was significantly higher than that of other three groups. It was verified that the dual-ligand modification on the POs was performed. Further investigation of material properties can show the vesicles to possess intracellular targeting properties. The ligandsmodified POs can be binding to the cell surface by means of receptor-specific interaction and rapidly taken up by the cells, most probably by the endocytotic pathway. ${ }^{35}$

\section{Cellular uptake of Tf/Tet-I-POs}

The cellular uptake results indicated that highest uptake was found for 6-coumarin-Tf/Tet-1-POs in bEnd.3 cells, in contrast to 6-coumarin-POs group, where the fluorescence intensity was lowest (Figure 4). There was no significant difference between 6-coumarin-Tf/Tet-1-POs and 6-coumarin$\mathrm{Tf} /$-POs groups in the fluorescence intensity. These results suggest that Tf-modified POs are capable of actively targeting the BBB. In the cell monolayer permeability test (Figure 4I), 6-coumarin-Tf/Tet-1-POs passed through the BCECs, and then the uptake of neuro- 2 cells increased, which differed significantly from the other three groups $(P<0.05)$. There was a significant difference between 6-coumarin-Tf/ Tet-1-POs and 6-coumarin-Tf/-POs groups $(P<0.05)$. The 6-coumarin-Tf/Tet-1-POs group and 6-coumarin-Tet-1-POs group also had a significant difference $(P<0.05)$. These results suggest that Tet-1-modified POs can actively target neurons; moreover, the dual-ligands-modified POs have better brain-targeting property than that of single-ligandmodified POs.

Table I Various components (\%) of CIs, OIs, and NIs of surface layer of POs were estimated using XPS analysis

\begin{tabular}{|c|c|c|c|c|c|c|c|c|c|}
\hline \multirow[t]{4}{*}{ Samples } & \multicolumn{3}{|c|}{ Elemental ratio (\%) } & \multicolumn{3}{|c|}{ CIs envelope ratios (\%) } & \multicolumn{3}{|c|}{ Ols envelope ratios (\%) } \\
\hline & \multirow[t]{3}{*}{ C } & \multirow[t]{3}{*}{0} & \multirow[t]{3}{*}{$\mathbf{N}$} & C-C/C-H & C-O-C & $\mathrm{O}=\mathrm{C}-\mathrm{O}-\mathrm{C}$ & O-C $=\mathbf{O}$ & $\mathbf{O}=\mathbf{C}$ & O-C \\
\hline & & & & \multicolumn{6}{|c|}{ Binding energy (eV) } \\
\hline & & & & 285.1 & 286.4 & 287.6 & 289.1 & 532.6 & 533.4 \\
\hline POs & 65.4 & 34.3 & 0.00 & 44.1 & 26.3 & 17.9 & 11.7 & 42.1 & 57.9 \\
\hline Tf-POs & 65.5 & 34.0 & 0.46 & 43.0 & 26.6 & 18.2 & 12.2 & 43.0 & 57.0 \\
\hline Tet-I-POs & 65.2 & 33.5 & 0.67 & 45.4 & 25.9 & 18.5 & 10.2 & 42.9 & 57.1 \\
\hline Tf/Tet-I-POs & 63.9 & 34.2 & 0.90 & 44.0 & 26.2 & 18.1 & 11.7 & 44.1 & 55.9 \\
\hline
\end{tabular}

Abbreviations: POs, polymersomes; XPS, X-ray photoelectron spectroscopy; Tf, transferrin; CIs, carbon; OIs, oxygen; NIs, nitrogen. 


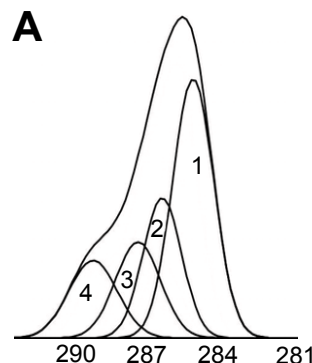

E

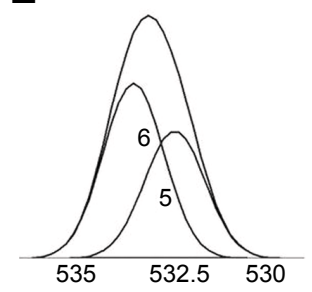

I

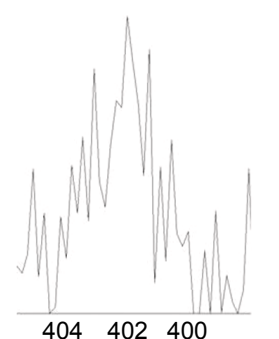

B

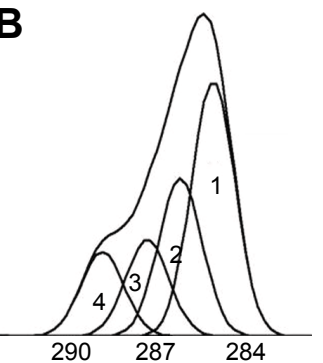

F

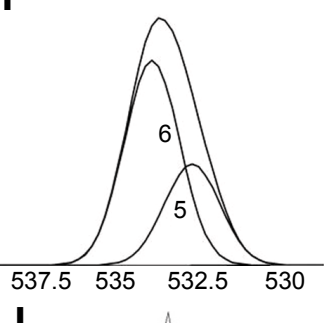

J

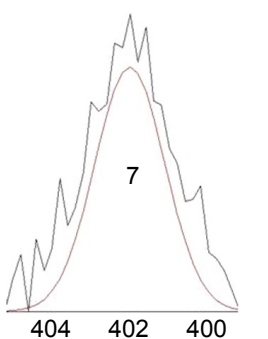

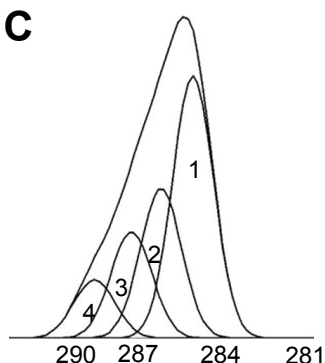

G

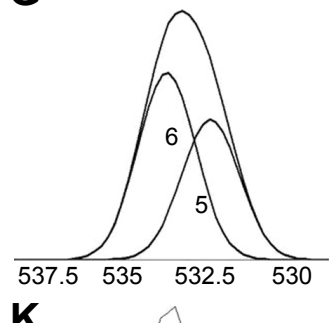

H
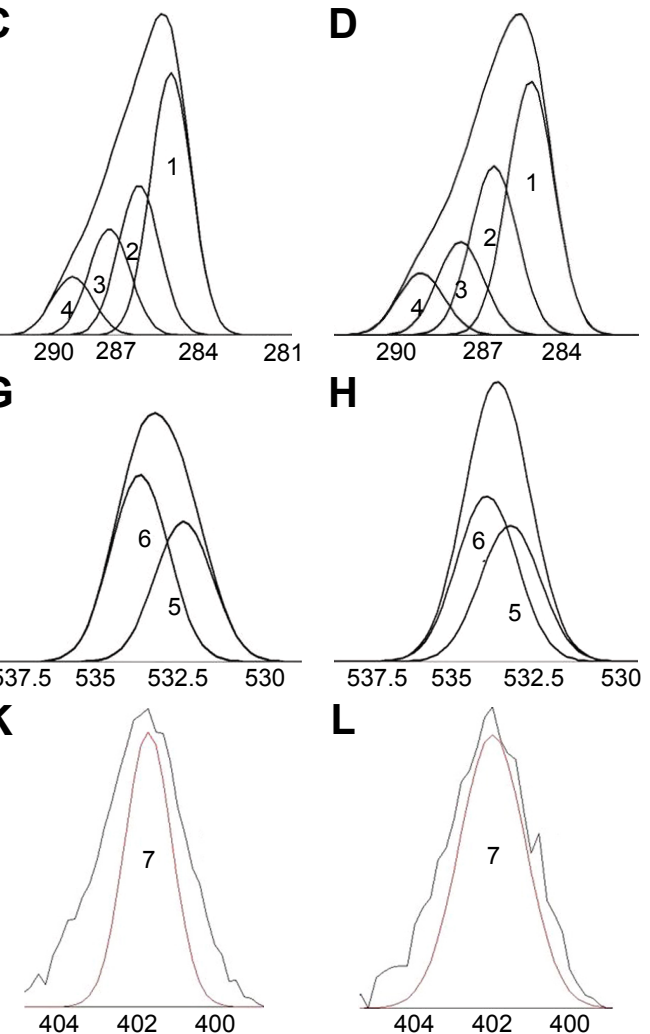

$\mathbf{L}$

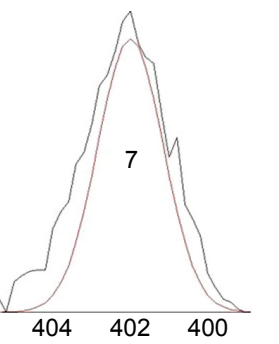

Figure 3 X-ray photoelectron spectroscopy analysis of POs modified with various ligands.

Notes: Carbon Cls envelopes: (A) POs, (B) Tf-POs, (C) Tet-I-POs, and (D) Tf/Tet-I-POs. Oxygen OIs envelopes: (E) POs, (F) Tf-POs, (G) Tet-I-POs, and (H) Tf/Tet-IPOs. Nitrogen envelopes: (I) POs, (J) Tf-POs, (K) Tet-I-POs, and (L) Tf/Tet-I-POs. The peaks at binding energies of 28I-307 eV, 527-545 eV, and 398-4I4 eV were ascribed to the elements $\mathrm{CIs}$, OIs, and NIs, respectively. The XPS survey scans were elucidated. The curve fitting results showed that the $\mathrm{Cls}$ regions could be presented by four peaks (A-D), of which the peak I signal represented C-C/C-H (285.I eV), the peak 2 signal represented C-O-C (286.4 eV), the peak 3 signal represented $\mathrm{O}=\mathrm{C}-\mathrm{C} *(-\mathrm{C})-\mathrm{O}$ $(287.6 \mathrm{eV})$, and peak 4 signal that corresponded to $\mathrm{O}-\mathrm{C}=\mathrm{O}(289 . \mathrm{l} \mathrm{eV})$, respectively. The $\mathrm{O}$ Is regions could be presented by peak signals that corresponded to $\mathrm{O}=\mathrm{C}$ (peak 5 , $532.6 \mathrm{eV}$ ) and O-C (peak 6, $533.4 \mathrm{eV})(\mathbf{E}-\mathbf{H})$. The NIs generated in Tf-POs, Tet-I-POs, and Tf/Tet-I-POs could be corresponded to peak 7.

Abbreviations: POs, polymersomes; Tf, transferrin.

\section{In vivo distribution of Tf/Tet-I-POs}

Figure 5 shows that there was almost no fluorescence distribution in the mice brain after iv injection of POs. Tf/ Tet-1-POs group had the strongest fluorescence, and the fluorescence intensity of Tf-POs and Tet-1-POs group was lower than that of $\mathrm{Tf} / \mathrm{Tet}-1-\mathrm{POs}$ group, proving that $\mathrm{Tf} / \mathrm{Tet}-$ 1-POs have good targeting effects.

\section{Pharmacokinetics}

The curcumin-loading efficiency of the POs was $2.54 \% \pm 0.16 \%$, and the encapsulation efficiency was $\sim 60.83 \% \pm 5.21 \%$. The choice of dissolution medium and the sink conditions has always been a problem for the low-solubility drugs like curcumin. For such compounds, release medium containing a surfactant or solvent in which the drug is soluble may be
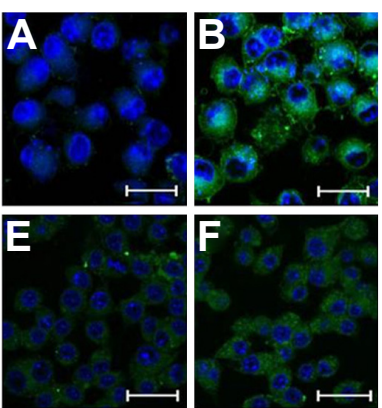
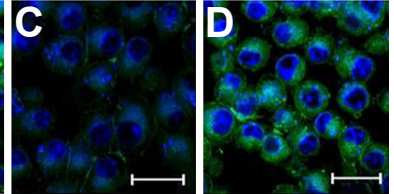

G
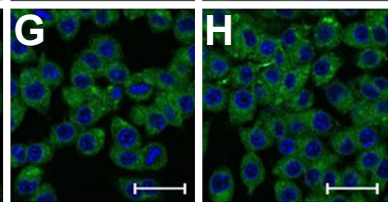

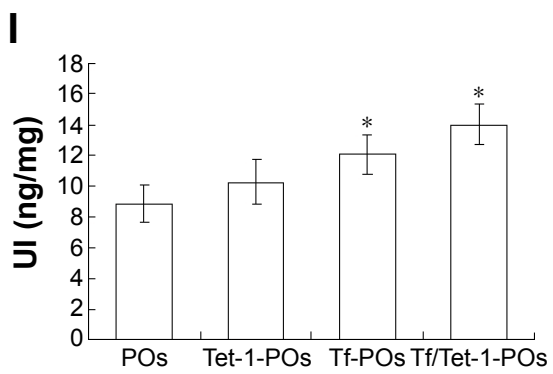

Figure 4 Cellular uptakes of various ligands-modified POs by bEnd. 3 and Neuro-2a cells at $37^{\circ} \mathrm{C}$ for 2 hours (bar $\left.200 \mu \mathrm{m}\right)$.

Notes: Fluorescence imaging of Neuro-2a cells: (A) 6-coumarin-POs, (B) 6-coumarin-Tf-POs, (C) 6-coumarin-Tet-I-POs, and (D) 6-coumarin-Tf/Tet-I-POs. Fluorescence imaging of BCECs: (E) 6-coumarin-POs, (F) 6-coumarin-Tf-POs, (G) 6-coumarin-Tet-I-POs, and (H) 6-coumarin-Tf/Tet-I-POs. (I) Uptake index of various ligands-modified POs by Neuro-2a cells in the coculture model of bEnd. 3 cells with Neuro-2a cells. $* P<0.05$.

Abbreviations: POs, polymersomes; Tf, transferrin; BCECs, brain capillary endothelial cells; UI, uptake index. 

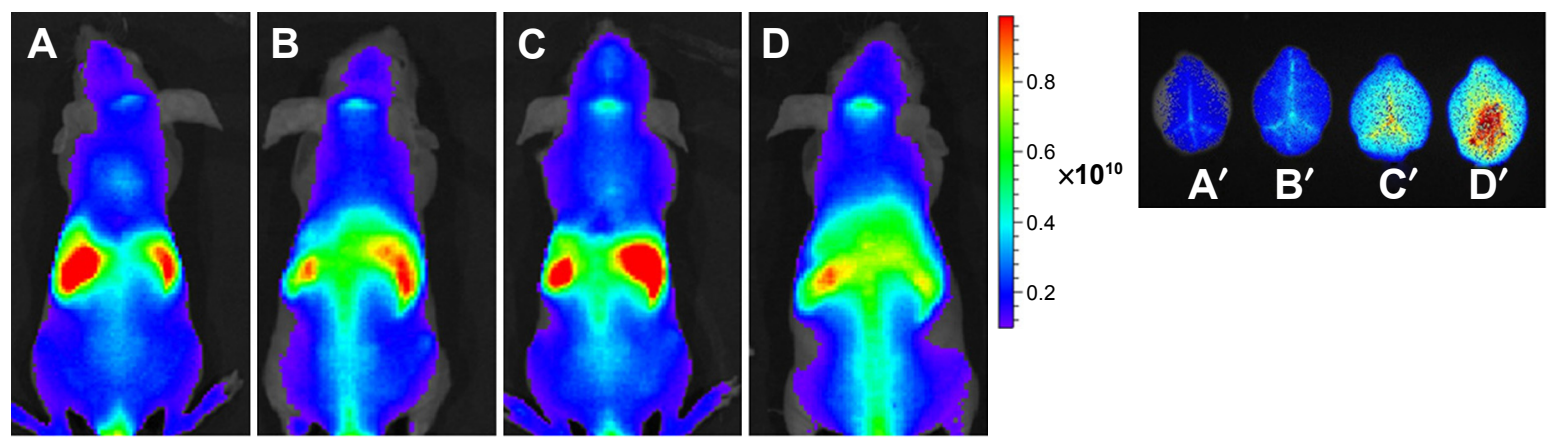

Figure 5 In vivo images.

Notes: In vivo images of nude mice administered with fluorescent probe DiR-labeled POs at 2 hours. (A) DiR-POs, (B) DiR-Tet-I-POs, (C) DiR-POs, and (D) DiR-Tf/Tet-

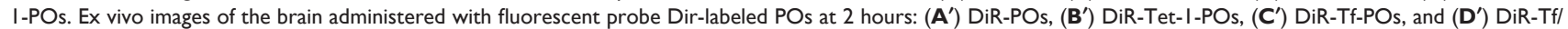
Tet-I-POs.

Abbreviations: DiR, I, I'-dioctadecyl-3,3,3',3'-tetramethylindotricarbocyanine iodide; POs, polymersomes; Tf, transferrin.

used to improve the solubility and dissolution rate. In this study, considering the solubility of curcumin, a $50 \%(\mathrm{v} / \mathrm{v})$ ethanol solution served as release medium. In vitro, the accumulated release percentage of drug was $\sim 75.69 \% \pm 4.82 \%$ after 72 hours (Figure 6). Pharmacokinetic parameters and brain-targeting properties were estimated in mice after iv injection. It indicated that the permeability-surface area $(P S)$ products all increased in the three experimental groups modified with targeting ligands, compared with the curcumin-POs group. The $P S$ of the curcumin-Tf/ Tet-1-POs was $0.28 \pm 0.05 \mathrm{~mL} / \mathrm{h} / \mathrm{g}$; it was, respectively, 1.72 and 5.20 times more than that of curcumin-Tf-POs group and curcumin-Tet-1-POs group. The highest $P S$ and accumulation were obtained in this group (Figure 7). The curcumin-Tf-POs and curcumin-Tf/Tet-1-POs groups were significantly different from the controls $(P<0.05)$. The brain tissue uptake rate $(\% \mathrm{ID} / \mathrm{g})$ of curcumin-Tf/Tet-1-POs group was $0.08 \pm 0.003$, which was 1.89 and 3.11 times more than that of curcumin-Tf-POs group and curcumin-Tet-1-POs group, respectively. It was also significantly different from

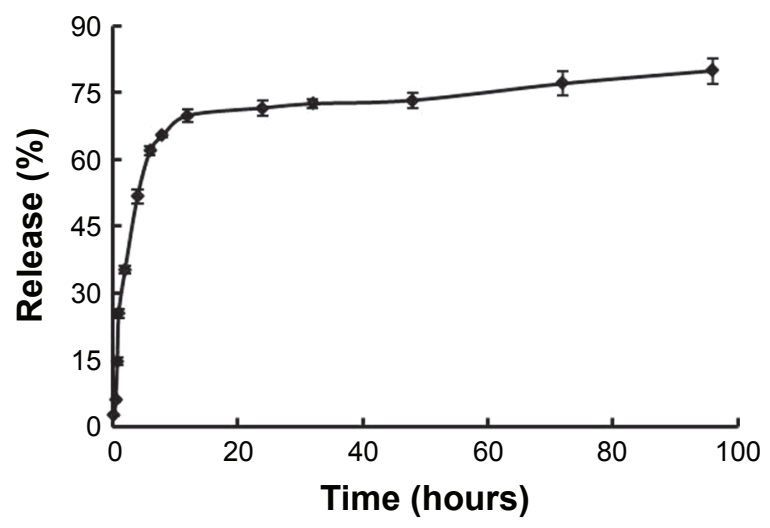

Figure 6 In vitro release of curcumin from Tf/Tet-I-POs $(n=3)$. Abbreviations: Tf, transferrin; POs, polymersomes. the POs group $(P<0.05)$. These results suggested that the brain transport efficiency was enhanced in combination with the Tf- and Tet-1-modified POs. Pharmacokinetic studies indicated that both BBB PS product and brain \% ID/g in the Tf/Tet-1-POs group were significantly higher than those of the control groups. Furthermore, combined with in vivo fluorescence imaging results of the animals, it can be observed that POs modified by Tf and Tet- 1 can increase the brain transport efficiency.

\section{Tf/Tet-I-POs loaded with curcumin ameliorated cognitive dysfunction induced by amyloid- $\beta_{1-42}$}

The Morris water maze results showed that with prolonged days of training, the sham control group showed a significantly shorter latency, whereas the AD control group did not demonstrate a significantly shortened latency; during the training period, the latency of the $\mathrm{AD}$ controls was significantly longer than that of the sham controls $(P<0.01)$ (Figure 8). Therefore, it is feasible to simulate that the cognitive dysfunction was induced by amyloid- $\beta_{1-42}$ injection in mice. The curcumin solution group had a long latency as well as no obvious difference compared with the $\mathrm{AD}$ controls $(P>0.05)$. The latency time of curcumin-POs group did not show a significant decrease with increased days of training.

The latency time of curcumin-Tf/Tet-1-POs group was significantly longer than the $\mathrm{AD}$ controls $(P<0.05)$ from the third day and was highly significantly different $(P<0.05)$ on the fifth day. On the sixth day of the probe trial conducted to test spatial memory, it was showed that the times of successful platform crossing were much lower for animals in the AD control group than in the sham controls. The times of platform crossing of the curcumin-Tf/Tet-1-PO and 

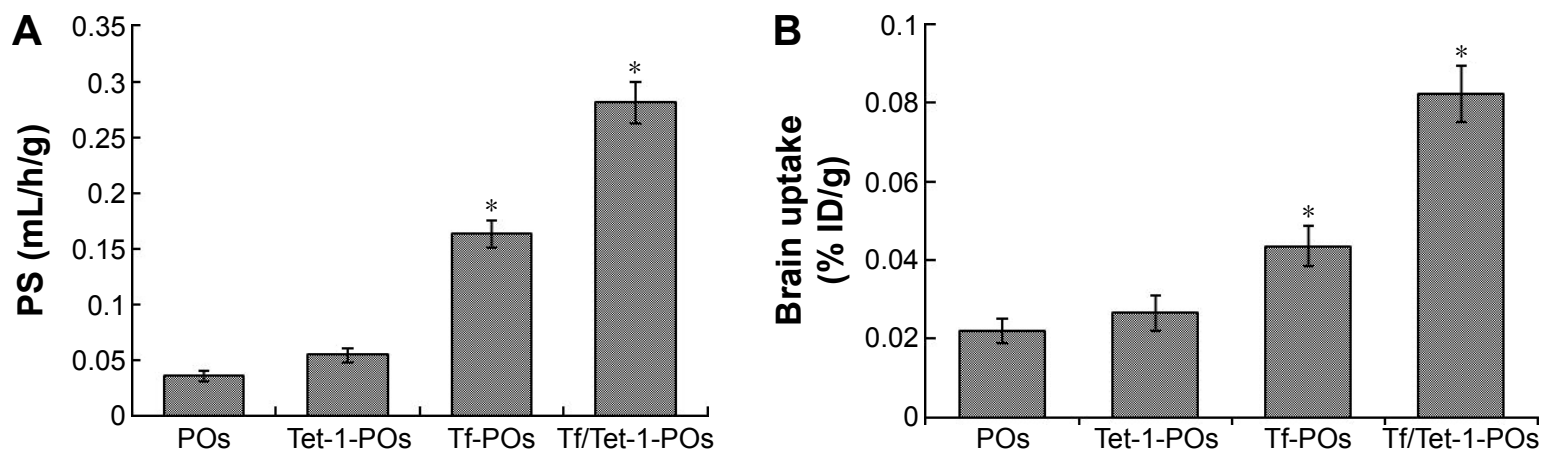

Figure 7 Pharmacokinetic parameters and brain transport properties of various ligands-modified POs loaded with curcumin administered by iv injection to mice ( $\mathrm{n}=6$ ). Notes: (A) BBB permeability-surface efficiency (PS). (B) Brain tissue uptake rate (\% ID/g brain). PS refers to the brain permeability surface area product. $* P<0.05$ values indicate the difference as compared with the POs control group.

Abbreviations: POs, polymersomes; iv, intravenous; BBB, blood-brain barrier; Tf, transferrin.

curcumin-Tf-PO groups were all significantly more than that of the sham group $(P<0.01)$. The crossing times of animals in the curcumin-Tf/Tet-1-PO group were more than the Tf/ Tet-1-PO group $(P<0.05)$. Curcumin-Tf/Tet-1-POs had significant effects in improving $A \beta$-induced memory and cognitive impairment in mice.

The therapeutical potential of herbs and marine natural products plays a role in prevention and cure of neurodegenerative diseases. Some phytocompounds, such as antioxidant and flavonoid-rich natural products, may hinder neurodegeneration, and improve memory and cognitive function. Kurapati et al described the neuroprotective effects of extract of ashwagandha against $\beta$-amyloid-induced toxicity. ${ }^{36}$ Curcumin plays a critical role in antioxidation and anti-inflammation and prevents the aggregation of $A \beta$ by inhibiting the activities of nuclear factors, lipoxygenase, and cyclooxygenase- $2 .{ }^{6}$
Some studies have shown that curcumin can bind to $A \beta$ and inhibit the formation of neurotoxic aggregates, so it destabilizes $\beta$-pleated sheet and leads to the reformation of $A \beta$ monomers. ${ }^{67}$ However, the potential therapeutic applications of curcumin were limited by its low bioavailability and a short biological half-life; there also has occurred weak transportation across the BBB via iv administration. ${ }^{8,10}$ The curcumin had been entrapped in POs that enhanced drug stability in the blood, and improved curcumin transport into the brain. Compared with the control group, the learning and memory ability of curcumin-Tf/Tet-1-POs group was significantly superior to that of the controls after the third day and was also stronger than that of the Tet-1-POs group. Curcumin-Tf/ Tet-1-POs could transport curcumin to the brain with a higher targeting efficiency and had a better therapeutic effect on the $\mathrm{A} \beta$-induced $\mathrm{AD}$ model.
A

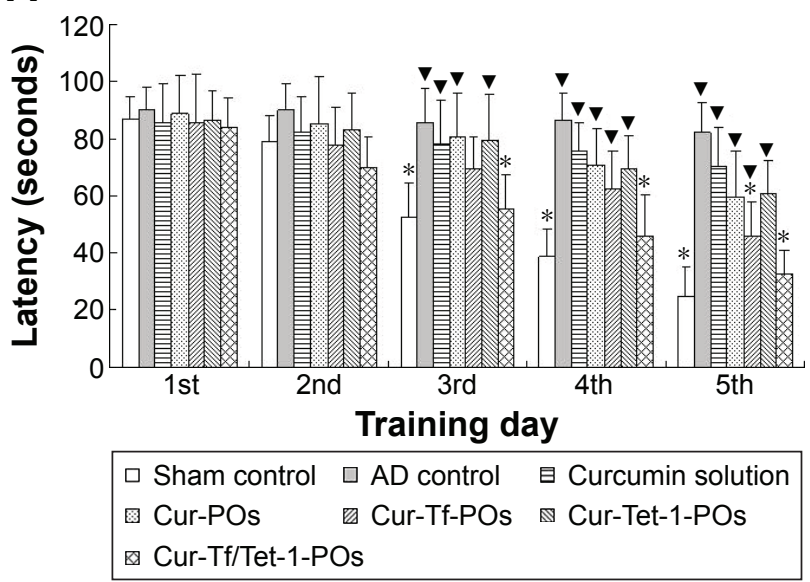

B

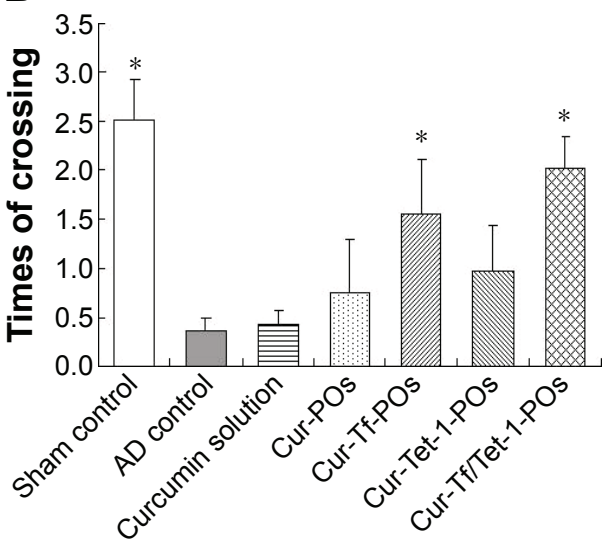

Figure 8 Neuroprotection offered by various ligands-modified POs loaded with curcumin against amyloid- $\beta_{1-42}$-induced cognitive dysfunction in mice estimated during the Morris water maze test.

Notes: (A) Behavioral test procedures performed in the first 5 days. (B) A spatial probe trial carried out on the sixth day. Sham control: saline was injected instead of amyloid- $\beta_{1-42}$; AD control: intravenous administration of saline was carried out. $\nabla P<0.05$, significant difference compared with the sham control; $* P<0.05$, significant difference compared with the AD control $(n=8)$.

Abbreviations: POs, polymersomes; AD, Alzheimer's disease; Cur, curcumin; Tf, transferrin. 
Neuropathological features of AD include amyloid plaques, neurofibrillary tangles, and loss of synapses and neurons. Overexpression and aberrant splicing of APP that results in abnormal deposits of insoluble $A \beta$ peptide is considered to be the major incentive for the pathological changes of $\mathrm{AD} \cdot{ }^{37,38}$ The toxic $\mathrm{A} \beta$ fragment, amyloid- $\beta_{1-42}$, exerts neurotoxicity on rat hippocampal neurons in primary culture. ${ }^{31}$ Directly injecting amyloid- $\beta_{1-42}$ into the hippocampus of rats can induce characteristic pathological changes of AD. ${ }^{30}$ Behavioral test is commonly used for the evaluation of memory and cognitive function. The Morris water maze test has been developed and used to assess related forms of spatial learning and memory. In terms of information processing and abstraction, the storage mechanism of this spatial reference memory is mainly related to the brain limbic system, which includes the hippocampus and related cerebral cortices. Declarative memory is first impaired in $\mathrm{AD}$ patients. Therefore, for the detection of memory attributes, it is appropriate to use the Morris water maze to screen effective medicines for the treatment of such diseases. ${ }^{39}$

\section{Conclusion}

In this study, dual-ligands-modified POs were prepared as a novel brain-targeting vector. Due to the coupling of Tf with the TfRs on BCECs, the transport of drug by the delivery system into the brain was increased. Moreover, Tet-1 peptide possesses a high affinity for the GT1B receptors on neurons, promoting two stages of brain targeting. Finally, POs functionalized by Tf and Tet-1 peptide transported the curcumin into brain and ameliorated cognitive dysfunction induced by amyloid- $\beta_{1-42}$ in mice. This brain delivery system facilitated the local release and more efficient delivery of therapeutics to the central nervous system; therefore, it can be applied to multiple vehicles for treating neurodegenerative diseases.

\section{Acknowledgments}

This work was supported by National Natural Science Foundation of China (81302714) and Innovation Program of Shanghai Municipal Education Commission (15ZZ041).

\section{Disclosure}

The authors report no conflicts of interest in this work.

\section{References}

1. Discher DE, Eisenberg A. Polymer vesicles. Science. 2002;297(5583): 967-973.

2. Lee JS, Feijen J. Polymersomes for drug delivery: design, formation and characterization. J Control Release. 2012;161(2):473-483.
3. Discher BM, Won YY, Ege DS, et al. Polymersomes: tough vesicles made from diblock copolymers. Science. 1999;284(5417):1143-1146.

4. Wortmann M. Dementia: a global health priority-highlights from an ADI and world health organization report. Alzheimers Res Ther. 2012; 4(5):40.

5. Hardy J. The amyloid hypothesis for Alzheimer's disease: a critical reappraisal. J Neurochem. 2009;110(4):1129-1134.

6. Aggarwal BB, Sung B. Pharmacological basis for the role of curcumin in chronic diseases: an age-old spice with modern targets. Trends Pharmacol Sci. 2009;30(2):85-94.

7. Lim GP, Chu T, Yang F, et al. The curry spice curcumin reduces oxidative damage and amyloid pathology in an Alzheimer transgenic mouse. J Neurosci. 2001;21(21):8370-8377.

8. Pak Y, Patek R, Mayersohn M. Sensitive and rapid isocratic liquid chromatography method for the quantitation of curcumin in plasma. $J$ Chromatogr B Analyt Technol Biomed Life Sci. 2003;796(2):339-346.

9. Ono K, Naiki H, Yamada M. The development of preventives and therapeutics for Alzheimer's disease that inhibit the formation of betaamyloid fibrils (fAbeta), as well as destabilize preformed fAbeta. Curr Pharm Des. 2006;12(33):4357-4375.

10. Sun M, Su X, Ding B, et al. Advances in nanotechnology-based delivery systems for curcumin. Nanomedicine (Lond). 2012;7(7):1085-1100.

11. Misra R, Sahoo SK. Coformulation of doxorubicin and curcumin in poly(D,L-lactide-co-glycolide) nanoparticles suppresses the development of multidrug resistance in K562 cells. Mol Pharm. 2011;8(3): 852-866.

12. Stamatovic SM, Keep RF, Andjelkovic AV. Brain endothelial cell-cell junctions: how to "open" the blood brain barrier. Curr Neuropharmacol. 2008;6(3):179-192.

13. Pardridge WM. Drug and gene targeting to the brain with molecular Trojan horses. Nat Rev Drug Discov. 2002;1(2):131-139.

14. Paris-Robidas S, Emond V, Tremblay C, et al. In vivo labeling of brain capillary endothelial cells after intravenous injection of monoclonal antibodies targeting the transferrin receptor. Mol Pharmacol. 2011;80(1): 32-39.

15. Pang Z, Gao H, Yu Y, et al. Enhanced intracellular delivery and chemotherapy for glioma rats by transferrin-conjugated biodegradable polymersomes loaded with doxorubicin. Bioconjug Chem. 2011;22(6): $1171-1180$.

16. Chen C, Fu Z, Kim JJ, Barbieri JT, Baldwin MR. Gangliosides as high affinity receptors for tetanus neurotoxin. J Biol Chem. 2009;284(39): 26569-26577.

17. Townsend SA, Evrony GD, Gu FX, et al. Tetanus toxin C fragmentconjugated nanoparticles for targeted drug delivery to neurons. Biomaterials. 2007;28(34):5176-5184.

18. Kwon EJ, Lasiene J, Jacobson BE, et al. Targeted nonviral delivery vehicles to neural progenitor cells in the mouse subventricular zone. Biomaterials. 2010;31(8):2417-2424.

19. Liu JK, Teng Q, Garrity-Moses M, et al. A novel peptide defined through phage display for therapeutic protein and vector neuronal targeting. Neurobiol Dis. 2005;19(3):407-418.

20. Bhattacharyya S, Khan JA, Curran GL, et al. Efficient delivery of gold nanoparticles by dual receptor targeting. Adv Mater. 2011;23(43): 5034-5038.

21. Gao HL, Pan SQ, Yang Z, et al. A cascade targeting strategy for brain neuroglial cells employing nanoparticles modified with angiopep-2 peptide and EGFP-EGF1 protein. Biomaterials. 2011;32(33):8669-8675.

22. Yu Y, Pang Z, Lu W, et al. Self-assembled polymersomes conjugated with lactoferrin as novel drug carrier for brain delivery. Pharm Res. 2012;29(1):83-96.

23. Li XT, Ju RJ, Li XY, et al. Multifunctional targeting daunorubicin plus quinacrine liposomes, modified by wheat germ agglutinin and tamoxifen, for treating brain glioma and glioma stem cells. Oncotarget. 2014; 5(15):6497-6511.

24. Wu J, Zhao J, Zhang B, et al. Polyethylene glycol-polylactic acid nanoparticles modified with cysteine-arginine-glutamic acid-lysine-alanine fibrin-homing peptide for glioblastoma therapy by enhanced retention effect. Int J Nanomedicine. 2014;9:5261-5271. 
25. Douville NJ, Tung YC, Li R, et al. Fabrication of two-layered channel system with embedded electrodes to measure resistance across epithelial and endothelial barriers. Anal Chem. 2010;82(6):2505-2511.

26. Chereddy KK, Coco R, Memvanga PB, et al. Combined effect of PLGA and curcumin on wound healing activity. J Control Release. 2013; 171(2):208-215

27. Gao Y, Li Z, Sun M, et al. Preparation, characterization, pharmacokinetics, and tissue distribution of curcumin nanosuspension with TPGS as stabilizer. Drug Dev Ind Pharm. 2010;36(10):1225-1234.

28. Huwyler J, Wu DF, Pardridge WM. Brain drug delivery of small molecules using immunoliposomes. PNAS. 1996;93:14164-14169.

29. Pang ZQ, Lu W, Gao HL, et al. Preparation and brain delivery property of biodegradable polymersomes conjugated with OX26. J Control Release. 2008;128:120-127.

30. Lee HE, Kim DH, Park SJ, et al. Neuroprotective effect of sinapic acid in a mouse model of amyloid $\beta(1-42)$ protein-induced Alzheimer's disease. Pharmacol Biochem Behav. 2012;103(2):260-266.

31. Brouillette J, Caillierez R, Zommer N, et al. Neurotoxicity and memory deficits induced by soluble low-molecular-weight amyloid- $\beta_{1-42}$ oligomers are revealed in vivo by using a novel animal model. J Neurosci. 2012;32(23):7852-7861.

32. Moon M, Choi JG, Nam DW, et al. Ghrelin ameliorates cognitive dysfunction and neurodegeneration in intrahippocampal amyloid- $\beta_{1-42}$ oligomer-injected mice. J Alzheimers Dis. 2011;23(1):147-159.
33. Yu Y, Jiang X, Gong S, et al. The proton permeability of self-assembled polymersomes and their neuroprotection by enhancing a neuroprotective peptide across the blood-brain barrier after modification with lactoferrin. Nanoscale. 2014;6(6):3250-3258.

34. Vorhees CV, Williams MT. Morris water maze: procedures for assessing spatial and related forms of learning and memory. Nat Protoc. 2006; $1(2): 848-858$

35. BrozP, Benito SM, Saw C, etal. Cell targeting by a generic receptor-targeted polymer nanocontainer platform. J Control Release. 2005;102(2): 475-488.

36. Kurapati KR, Atluri VS, Samikkannu T, et al. Ashwagandha (Withania somnifera) reverses $\beta$-amyloid ${ }_{1-42}$ induced toxicity in human neuronal cells: implications in HIV-associated neurocognitive disorders (HAND). PLoS One. 2013;8(10):1-15.

37. Serrano-Pozo A, Frosch MP, Masliah E, Hyman BT. Neuropathological alterations in Alzheimer disease. Cold Spring Harb Perspect Med. 2011;1(1):1-23.

38. Bidros DS, Vogelbaum MA. Novel drug delivery strategies in neurooncology. Neurotherapeutics. 2009;6(3):539-546.

39. Bromley-Brits K, Deng Y, Song W. Morris water maze test for learning and memory deficits in Alzheimer's disease model mice. J Vis Exp. $2011 ; 20 ;(53): 1-5$.
International Journal of Nanomedicine

\section{Publish your work in this journal}

The International Journal of Nanomedicine is an international, peerreviewed journal focusing on the application of nanotechnology in diagnostics, therapeutics, and drug delivery systems throughout the biomedical field. This journal is indexed on PubMed Central, MedLine, CAS, SciSearch $\AA$, Current Contents $₫ /$ Clinical Medicine,

\section{Dovepress}

Journal Citation Reports/Science Edition, EMBase, Scopus and the Elsevier Bibliographic databases. The manuscript management system is completely online and includes a very quick and fair peer-review system, which is all easy to use. Visit http://www.dovepress.com/ testimonials.php to read real quotes from published authors. 